\title{
Parâmetros e métodos adotados no regulamento de etiquetagem da eficiência energética de edifícios - parte 2: método de simulação
}

\author{
Parameters and methods adopted in the energy efficiency \\ labelling regulation for buildings - part 2: simulation \\ method
}

\section{Joyce Correna Carlo Roberto Lamberts}

\section{Resumo \\ A}

descrição dos métodos de avaliação do regulamento de eficiência energética de edifícios do Programa Brasileiro de Etiquetagem está contida em dois artigos. A primeira parte apresenta e discute o método prescritivo. A segunda parte refere-se ao método de simulação e suas aplicações, em que são discutidas questões acerca da simulação envolvendo o desempenho do edifício condicionado e o uso da simulação de ventilação natural, mas com enfoque no entorno do edifício condicionado e o aproveitamento da luz natural. Para tanto, um edifício real foi simulado de acordo com o método do Regulamento Técnico da Qualidade do Nível de Eficiência Energética de Edifícios Comerciais, de Serviços e Públicos (RTQ-C), com e sem sombreamento do entorno e com e sem brises. Seu modelo de referência foi também simulado, com e sem sombreamento do entorno. Os resultados de consumo anual foram comparados para avaliar o potencial de economia de eletricidade de cada modelo em relação a duas opções de modelos de referência. Essas duas opções foram

Joyce Correna Carlo Departamento de Arquitetura e Universidade Federal de Viçosa Avenida Peter Henry Rolfs, $\mathrm{s} / \mathrm{n}$ Viçosa - MG - Brasil CEP 36570-000 Tel.: (31) 3899-1982 Email: joycecarlo@ufv.br

Roberto Lamberts Laboratório de Eficiência Energética em Edificações Departamento de Engenharia Universidade Federal de Santa Catarina Cx. Postal 476

Florianópolis - SC - Brasil CEP $88040-900$

Tel.: (48) 3721-5184

lamberts@labeee.ufsc.br

Recebido em 06/01/2010

Aceito em 24/04/2010 também discutidas, mostrando por que o modelo de referência não deve possuir sombreamento no entorno para a simulação, segundo o RTQ-C.

Palavras-chave: Eficiência energética. Etiquetagem. Simulação.

\section{Abstract}

The description of the evaluation methods of the Brazilian energy efficiency regulations for buildings is presented in two papers. The first part presents and discusses the prescriptive method. The second part deals with the simulation method and its use, and discusses issues related to the simulation of the performance of air-conditioned buildings in particular, but also of naturally ventilated buildings, focusing on the surroundings of the air-conditioned building and the use of daylight. An existing building was simulated based on the method of the Technical Regulations for Energy Efficiency Labelling of Commercial Buildings $(R T Q-C)$ in Brazil, with and without surrounding shading, and with and without brise soleil. Its reference model was also simulated with and without surrounding shading. The results of annual electricity consumption were compared in order to assess the savings potential of each model in relation to the two reference models. The results of the two reference models were also discussed, explaining why the reference model should not have surrounding shading for simulation, according to the $R T Q-C$.

Keywords: Energy efficiency. Labelling. Simulation. 


\section{Introdução}

O uso da simulação em normas, regulamentos e programas de eficiência energética para edifícios é adotado em diversos países. Um dos regulamentos mais relevantes é a Norma 90.1 da ASHRAE (2007), que contém um método de simulação para seus requisitos mínimos, cujo cumprimento é obrigatório, e outro método informativo, em seu apêndice $\mathrm{G}$, para obtenção de economias acima do mínimo exigido pela norma.

O Regulamento Técnico da Qualidade do Nível de Eficiência Energética de Edifícios Comerciais, de Serviços e Públicos (RTQ-C) (BRASIL, 2009), lançado em fevereiro de 2009, incluiu simulação como um de seus métodos de avaliação para a obtenção da Etiqueta Nacional de Conservação de Energia (ENCE). O outro método é o prescritivo, baseado em tabelas e equações, cuja grande parte foi também desenvolvida por simulação.

Segundo Gonçalves e Almeida (1995), simulação é um processo de emulação da realidade, quando vista sob a ótica do usuário. Um especialista que domina a fundo a simulação tende a vê-la como "modelos matemáticos detalhados para previsão de alguns aspectos do desempenho de edificações" (HAVES, 2004). A simulação termoenergética lida com interações complexas sob uma diversidade de condições, aproximando-se das interações reais mais complexas ainda pertencentes aos edifícios. Assim, embora se aproxime da realidade do edifício, ainda lida com simplificações da realidade, representadas nos modelos. Um modelo pode ser definido como uma entidade que representa outra entidade, porém reduzida às características de interesse (MAHDAVI, 2004). No caso da simulação de edificações, o modelo é uma descrição numérica de uma edificação, porém reduzida às características intervenientes em seu desempenho térmico e energético. Essas características reúnem uma série de outros modelos numéricos envolvidos no processo, desde fenômenos físicos até a geometria do edifício e seu uso. Assim, essa simplificação possibilita a exploração, investigação e compreensão (MAHDAVI, 2004) dos fenômenos envolvidos com as trocas térmicas, o uso do edifício e, portanto, seu desempenho, refletido pelas cargas térmicas ou pelo consumo de energia.

Carlo (2008) mostrou que a eficiência energética de um edifício é avaliada comparando-se o edifício com ele próprio. Em outras palavras, os parâmetros intervenientes na eficiência energética têm impacto variado. Os parâmetros de maior impacto, chamados de características primárias, devem ser idênticos no edifício para viabilizar a comparação energética entre dois ou mais modelos, a fim de verificar qual é o mais eficiente. Além disso, a eficiência é temporal, visto que é baseada nos recursos tecnológicos da época. Assim, o RTQ-C propôs a avaliação por um método flexível que permite a análise comparativa da eficiência usando dois modelos de edifícios com características primárias comuns.

Características primárias em comum para os dois modelos não dispensam a necessidade de que alguns parâmetros referenciais sejam predefinidos, a fim de viabilizar a avaliação. Os parâmetros referenciais são baseados no método prescritivo, que exige atualização constante, visando manter os níveis de eficiência no patamar desejado, em concordância com as inovações tecnológicas de seu tempo.

Outros parâmetros predefinidos referem-se ao programa de simulação e ao método de modelagem. Embora o RTQ-C não exija um programa específico, a qualidade da simulação está atrelada a um método de avaliação do programa computacional, o método BESTEST (ASHRAE, 2004), indicado para edifícios condicionados. Ficam ainda de fora os recursos de ventilação e iluminação natural, ambos com grande potencial de aproveitamento em edifícios no Brasil, mas que ainda não apresentam métodos de avaliação para seus programas de simulação. Além disso, o regulamento apresenta um método de simulação que se refere ao que deve e pode ser modelado, tanto no modelo do edifício real quanto no modelo de referência. Alguns parâmetros relativos ao uso do edifício são de livre modelagem em ambos, como padrões de uso e cargas internas de equipamentos, contanto que sejam idênticos. Já outros devem seguir regras específicas, não apenas em relação ao exigido pelo modelo de referência, mas também em relação ao modelo do edifício proposto. Como exemplo, há procedimentos de modelagem quando somente a envoltória será avaliada e não existe projeto de iluminação ou de condicionamento de ar. Outro exemplo é a opção da equipe de projeto/proprietário/simulador de fazer uso do sombreamento do entorno. Esse recurso é válido somente no método de simulação e é opcional. Pode ser usado quando o edifício já é sombreado pelos edifícios do entorno, sendo, portanto, desnecessário o sombreamento por brises ou outro dispositivo equivalente. $\mathrm{Na}$ verdade, os edifícios do entorno são dispositivos equivalentes que apresentam a diferença de também sombrear as paredes. São os casos dos edifícios localizados em grandes centros comerciais, onde predomina uma paisagem urbana verticalizada. 
Este artigo apresenta os recursos do RTQ-C aplicados ao método de simulação ante as limitações do método prescritivo, visando discutir as questões relativas à simulação no regulamento. Em especial, discute-se o sombreamento do entorno usado exclusivamente no método de simulação. Além disso, o aproveitamento da luz natural foi somado à análise energética para enriquecer a discussão acerca do sombreamento do entorno, integrando a parcela térmica e luminosa na avaliação energética. ${ }^{1}$ Em paralelo, são discutidos os tipos de programas de simulação adotados em função dos pré-requisitos existentes no regulamento, com destaque aos parâmetros futuros que podem ser incorporados.

Para tanto, faz-se anteriormente uma revisão de partes de duas normas da ASHRAE, a Norma 90.1 (2007) e o Método BESTEST (2004), para embasar a explicação. Outras normas e regulamentos são citados ainda na fase de estudo.

Há outras limitações relacionadas ao regulamento e à simulação que não serão abordadas, mas merecem um destaque. A prática da simulação é recente pela academia e mais ainda pelo mercado da construção civil. A qualidade do simulador e os recursos dos programas que adota ainda serão discutidos por bastante tempo. A IBPSA-Brasil, Associação Internacional de Simuladores de Edifícios do Brasil, inicia a discussão, que também se estende pela ASHRAE americana com vistas a uma futura certificação dos simuladores. Por enquanto, o RTQ-C aceita simulações de especialistas, desde que sejam garantidas a qualidade da simulação, sem, no entanto, entrar em qualquer tipo de capacitação.

\section{Simulação na Norma 90.1}

A Norma 90.1 da ASHRAE (2007), norma de eficiência energética para edifícios não residenciais e para edificações residenciais acima de dois pavimentos, apresenta dois métodos de uso da simulação. Visto se tratar de norma obrigatória de requisitos mínimos de eficiência, ela apresenta o Energy Cost Budget como método de simulação que compara o custo da energia de um edifício proposto com o custo da energia de um edifíciobase (budget building). A Norma 90.1 apresenta ainda um método informativo em seu apêndice $\mathrm{G}$, referente à simulação de edifícios que devem atingir maiores economias que o mínimo exigido pela norma. Nesse caso, o baseline é o edifíciobase cujo custo da energia deve ser comparado ao

\footnotetext{
${ }^{1} \mathrm{O}$ aproveitamento da luz natural está inserido na avaliação pelo método prescritivo nas bonificações. No método de simulação, ele deve ser simulado em conjunto com a parcela termoenergética.
}

custo da energia do modelo do edifício proposto. $\mathrm{O}$ custo da energia do edifício proposto deve ser menor que o custo do budget building no primeiro caso e, no segundo, o custo da energia do edifício proposto é quantificado percentualmente em relação ao custo da energia do budget building.

$\mathrm{O}$ budget building deve ser modelado de maneira idêntica ao modelo do edifício proposto, exceto pelos parâmetros fixados na norma, como: janelas, que devem ser no máximo de $40 \%$ da área de fachada; cores, cuja absortância máxima é 0,30 ; potência do sistema de iluminação, que deve atender a valores tabelados; ou sistema de condicionamento de ar, que deve ser um dos oito modelos de sistemas disponíveis para o método, cuja seleção irá depender do tipo de sistema e do uso presentes no edifício proposto.

Em ambos os casos - tanto para atender aos requisitos mínimos quanto para quantificar uma economia percentual em relação ao mínimo - o custo da energia em grandes edifícios é geralmente decorrente de um consumo que, por resfriamento, é baseado na energia elétrica, enquanto o consumo por aquecimento é baseado em combustíveis fósseis, como gás, carvão ou até diesel. Assim, usou-se um indicador monetário para integrar os consumos de fontes energéticas distintas. Esses dois usos finais, aquecimento e resfriamento, são comuns naquele país devido ao clima de suas regiões.

O clima também determina o método de simulação. Para qualquer ambiente condicionado, é exigida a modelagem do aquecimento e resfriamento, mesmo que somente um destes esteja projetado no sistema de condicionamento de ar que irá atender ao ambiente. O método, assim como a norma, não trata de ambientes naturalmente ventilados, apesar de determinar os ambientes semicondicionados que, de acordo com seu conceito, são função da natureza do condicionamento e do tipo de isolamento térmico que suas paredes, cobertura e piso apresentam. Assim, a norma exige procedimentos de simulação que são válidos para o clima extremo do país, ignorando as condições de ventilação natural, pouco comuns na maior parte de seu território.

Ainda, a norma apresenta exigências específicas para o programa de simulação. Deve ter sido avaliado pelo método BESTEST (ASHRAE, 2004), ser capaz de modelar variações horárias com um mínimo de 1.400 horas em um ano ${ }^{2}$ para todos os sistemas existentes (iluminação,

\footnotetext{
${ }^{2}$ Acredita-se serem 700 horas por estação, o que

corresponde a um mês. Assim, o programa deve ser capaz de modelar um mês do verão e um mês do inverno, totalizando 1.400 horas.
} 
ocupação, equipamentos, aquecimento de água, etc.), modelar um mínimo de 10 zonas térmicas e modelar ciclos economizadores do condicionamento de ar, entre outros recursos. Deve ainda produzir relatórios horários e fornecer diretamente o custo da energia pela simulação.

\section{Método BESTEST para edifícios condicionados versus iluminação e ventilação natural}

Judkoff e Neymark (2009) indicaram três métodos de validação de programas computacionais: validação empírica, verificação analítica e testes comparativos. O método BESTEST (Building Energy Simulation Test) é um método de avaliação comparativa de programas de simulação termoenergética para identificar possíveis diferenças causadas por modelos matemáticos distintos, limitações de modelagem, erros do simulador ou até erros de código de programação (ASHRAE, 2004).

Como exigido pelo RTQ-C, o EnergyPlus é aprovado pelo método BESTEST da Norma 140 (ASHRAE, 2004). Realiza simulações do desempenho energético anual do edifício por meio do método de balanço térmico, fornecendo consumos por uso final calculados de 15 em 15 minutos ou em intervalos de tempo ainda menores.

Esse método é válido tanto para o edifício condicionado quanto para o edifício sem condicionamento e sem ventilação natural. ${ }^{3}$ Não inclui, portanto, a simulação de ventilação natural para cálculos das trocas térmicas e de massa, que carece de método consolidado de validação em programas computacionais. Um método BESTEST está em desenvolvimento para validar programas de simulação de ventilação natural, como parte dos programas de Aquecimento Solar e Resfriamento e de Conservação de Energia em Edifícios e Comunidades da IEA - International Energy Agency (JUDKOFF, 2008). Está em avaliação mediante a verificação analítica, que consiste em comparar os resultados do programa de simulação com soluções analíticas conhecidas e comprovadas. Entre os programas avaliados estão o EnergyPlus e o Esp-r (JUDKOFF; NEYMARK, 2009), ambos baseados em modelos de rede. Enquanto esses métodos não se consolidam em uma norma, as poucas validações em programas de ventilação natural são realizadas independentemente, como Gu (2007), que realizou uma validação da simulação da ventilação natural

\footnotetext{
${ }^{3} \mathrm{O}$ método inclui a infiltração, que provoca perdas e ganhos térmicos, mas não considera as trocas de massa decorrentes dos fluxos de ar que atravessam o ambiente.
}

do EnergyPlus, mas que envolvia o condicionamento de ar e as trocas por infiltração sem a verificação da temperatura interna dos ambientes. Esse tipo de validação é insuficiente para as condições climáticas brasileiras, que necessitam dos métodos de ventilação ainda em desenvolvimento.

Assim como ocorre com a ventilação natural, programas de simulação de iluminação natural também não possuem normas nacionais ou internacionais de validação. Duas frentes estão em desenvolvimento para suprir essa demanda.

O relatório técnico da CIE (2006) indica parâmetros que podem vir a se tornar uma norma de validação de programas de simulação de iluminação. São casos experimentais e analíticos que devem ser modelados e simulados no programa para verificar sua precisão na simulação da iluminação, tanto natural quanto artificial. Os testes também podem ser usados para comparação de programas visando verificar qual apresenta os melhores recursos para um uso específico.

Um método BESTEST para validação empírica da simulação envolvendo sombreamento, iluminação natural e interações dessas cargas no edifício também faz parte da iniciativa da IEA, através dos programas de Aquecimento Solar e Resfriamento e de Conservação de Energia em Edifícios e Comunidades (JUDKOFF, 2008). Mediante experimentos em campo, foram identificados parâmetros que deveriam ser reproduzidos em simulação. Vale ressaltar que os experimentos de ganhos térmicos foram conduzidos na Suíça, e os de iluminação natural, nos EUA, ou seja, ambos submetidos às condições de céu do hemisfério norte (LOUTZENHISER; MANZ; MAXWELL, 2007; MANZ et al., 2006). Uma série de exercícios foram aplicados por pesquisadores de diversas origens para validação, que incluíram programas como EnergyPlus, DOE 2.1-E e Esp-r.

Enquanto métodos são desenvolvidos para validação desses programas de simulação (PEREIRA et al., 2007), deve-se avaliar individualmente a qualidade dos programas. Reinhart, Mardaljevic e Rogers (2006) afirmaram que as simulações dinâmicas são mais vantajosas que as simulações estáticas por considerarem as variações diárias e sazonais da luz natural decorrentes também dos eventos meteorológicos, geralmente irregulares.

O programa DAYSIM utiliza o programa Radiance (REINHART; HERKEL, 2000) para realizar a simulação do sistema de iluminação com aproveitamento da luz natural com e sem modelos comportamentais, de acordo com o nível de iluminância-limite fornecido pelo simulador. 
Fornece as cargas parciais de um sistema dimerizável para um ano completo em formato que pode ser exportado como padrão de uso para o programa EnergyPlus (CRAWLEY et al., 2001) após rápido tratamento dos arquivos de saída. Além disso, permite o uso de arquivos climáticos do tipo epw como entrada no programa, que é o formato padrão do EnergyPlus. Permite, portanto, o uso dos mesmos dados de entrada tanto no programa de simulação da luz natural quanto no programa de simulação termoenergética, sem a necessidade de tratamento dos dados ou conversão dos formatos.

Considera-se que o Radiance é um programa internacionalmente aceito como referencial para outros estudos envolvendo simulação computacional de iluminação natural e foi, portanto, considerado válido diante das limitações do modelo de luz natural do EnergyPlus. Embora Ramos e Ghisi (2008) tenham mostrado semelhanças entre o EnergyPlus e o DaySim, seus resultados somente se aproximaram por tais modelos dinâmicos terem sido comparados a modelos de simulação estática. Ao serem comparados entre si, percebeu-se um superdimensionamento nas simulações de iluminação no EnergyPlus em relação ao DaySim em 2007, confirmado por Ramos e Ghisi (2009). Além disso, o EnergyPlus contém apenas dois pontos de medição, enquanto o DaySim permite a inserção de uma rede de pontos. Essas características justificam o uso do DaySim como programa de simulação da luz natural para integração aos resultados obtidos com o EnergyPlus.

No presente trabalho, foram realizadas simulações envolvendo os recursos de condicionamento artificial do programa EnergyPlus compatibilizados com os recursos de iluminação do programa DaySim, visando obter o consumo anual de energia elétrica dos modelos de edifícios. Portanto, não estão incluídas simulações de ventilação natural, cujo objetivo seria obter, entre outras possibilidades, as temperaturas internas dos ambientes.

\section{Método de simulação do RTQ-C}

No RTQ-C, a simulação é o método mais completo para qualquer análise do desempenho térmico e/ou energético do edifício, proporcionando flexibilidade nas opções que visam à racionalização do consumo de energia, o que inclui o processo de projeto. A simulação permite o estudo nos casos de edificações com volumetrias, aberturas ou proteções solares mais complexas. Permite também a flexibilidade no projeto do condicionamento de ar, em que o projetista pode propor outras soluções - distintas das exigências de projeto para sistemas centrais de nível A -, que poderão alcançar elevados níveis de eficiência, de acordo com as especificidades de seu projeto. Por fim, permite a avaliação das condições passivas de condicionamento, como estratégias bioclimáticas envolvendo a ventilação natural ou forçada $e$ inércia térmica para aquecimento ou resfriamento.

O método exige alguns pré-requisitos para o programa de simulação e para o arquivo climático utilizado. Ele deve ser realizado para um ano completo, utilizando um programa aprovado pelo método BESTEST da Norma 140 (ASHRAE, 2004). A análise de variações horárias do edifício, desde sua carga térmica até a ocupação nos ambientes internos exigida pelo BESTEST, refletese no desempenho dos sistemas de iluminação, equipamentos, condicionamento de ar, entre outros. É necessário que o programa modele inércia térmica, permita a modelagem multizonas e seja capaz de determinar a capacidade do sistema de condicionamento de ar. Ao contrário do BESTEST, que exige que o programa apresente possibilidade mínima de simulação sazonal, o método do RTQ-C exige a simulação anual, envolvendo 8.760 horas do ano. Já o arquivo climático deve conter dados horários que atendam às necessidades da simulação, cujos formatos mais comuns no Brasil são TRY e TMY2 ${ }^{4}$. Para a simulação da ventilação natural e de outras estratégias bioclimáticas, é apenas exigido que o programa apresente os recursos necessários para sua simulação. No entanto, sem método de validação consolidado na comunidade internacional, nada foi exigido para validação do programa de simulação de ventilação natural ou de iluminação natural. Espera-se que, no futuro, uma nova versão do regulamento possa incluir exigências de validação dos demais recursos, se não internacionais, a partir de esforços nacionais visando à uniformidade de recursos mínimos que tais programas devem apresentar.

Atendidos os pré-requisitos, a avaliação é realizada por meio da comparação do consumo de dois modelos de edifícios: um modelo real, baseado no edifício que está sendo proposto para receber a etiqueta, e um modelo de referência, definido com base no método prescritivo, porém com algumas especificidades, como uso de vidros claros e sem qualquer tipo de sombreamento. O modelo de referência deve possuir ainda diversas

\footnotetext{
${ }^{4}$ Arquivos deste tipo podem ser encontrados na página do EREE - Energy Efficiency and Renweable Energy

(http://apps1.eere.energy.gov/buildings/energyplus/) no formatos TMY2 (compilações IWEC e SWERA), e do LabEEE (www.labeee.ufsc.br), nos formatos TRY (compilações LabEEE), totalizando cerca de 30 cidades brasileiras.
} 
características, que devem ser idênticas às do modelo do edifício real, como forma do edifício, número de zonas térmicas, padrões de uso, ocupação e cargas internas de equipamentos. Compara-se o consumo anual de eletricidade simulado dos dois modelos, devendo o consumo do modelo do edifício real ser menor que o do modelo de referência para o nível de eficiência pretendido. Dessa forma, obtém-se o nível de eficiência geral do edifício.

\section{A questão do sombreamento do entorno}

O RTQ-C permite que o entorno seja usado - mas somente na simulação - na avaliação do nível de eficiência do edifício. Para tanto, os edifícios do entorno devem ser inseridos no modelo real e somente neste, deixando livre de sombreamento o edifício de referência.

Há duas visões distintas sobre esse item, ambas corretas e baseadas no mesmo conceito, mas com enfoques diferentes, as quais serão discutidas aqui. Carlo (2008) mostrou que a eficiência energética de um edifício é comparativa: um edifício qualquer deve ter seu nível de eficiência comparado com o nível de um edifício semelhante, cujas características primárias são iguais, e as diferenças na eficiência são verificadas em função das características secundárias. ${ }^{5}$

A partir desse conceito, pode-se afirmar que dois edifícios equivalentes (contendo as diferenças descritas acima) devem ter a localização idêntica para avaliar seu nível de eficiência, o que inclui seu entorno. Essa afirmação é válida em qualquer caso de simulação, exceto em casos especiais, como na simulação para etiquetagem.

No RTQ-C, um edifício real é comparado a um edifício de referência. O entorno do edifício pode ser incluído na simulação do edifício real, mas não no modelo do edifício de referência. Estar-se-ia então comparando edifícios em diferentes condições?

O modelo computacional do edifício de referência - em particular aqui é discutida a envoltória - é construído a partir de parâmetros específicos do método prescritivo. Ou seja, se o método prescritivo permite a avaliação de edifícios com inúmeras características distintas, a envoltória do modelo de referência só pode ser uma, considerando um nível de eficiência e para cada volumetria específica - FF e FA.
Além disso, como o próprio nome diz, é um modelo de referência. Sendo assim, para cada volumetria, existe apenas um modelo de referência nível A, um modelo de referência nível B, e assim por diante. Eles são fixos para uma volumetria e clima.

Simplificadamente, esses modelos nasceram do método prescritivo, composto das equações para avaliação da envoltória e dos pré-requisitos. Um olhar mais a fundo identifica que as equações são uma regressão linear multivariada cuja variável "Y" são os consumos de energia de diversos modelos de edifícios comerciais simulados (CARLO; LAMBERTS, 2008). Todos esses foram modelados sem o sombreamento do entorno, mas vários contêm os sombreamentos das proteções solares e as transmitâncias das paredes distintas. Assim, as escalas de eficiência energética da envoltória foram definidas para edifícios sem entorno, já que as equações referem-se a esse tipo de edifício. Assim foram definidos os edifícios de referência.

Dessa forma, o sombreamento do entorno é utilizado para dispensar o edifício da instalação de sombreamento excessivo quando já possui sombreamento vizinho. É fato que essa situação benéfica pode se alterar com a demolição do edifício vizinho, mas, como o uso do sombreamento do entorno é opcional, o proprietário do edifício deve estar ciente de que pode perder a etiqueta caso os edifícios vizinhos sejam demolidos. De fato, o sombreamento excessivo pode prejudicar o nível de eficiência do edifício, principalmente quando o aproveitamento da luz natural para a economia no sistema de iluminação artificial está também incluído na simulação. A seguir, são mostrados testes com essa situação.

\section{Aplicação do método de simulação}

Um edifício real de Florianópolis foi escolhido para modelagem, baseando-se nos dados levantados por Santana (2006) para edifícios empresariais de escritórios. Simulou-se de acordo com o método de simulação do RTQ-C, usando o modelo de referência calculado segundo o método prescritivo e outro modelo representando o edifício real.

\footnotetext{
${ }^{5}$ Ou os edifícios podem conter todas as suas características primárias e secundárias idênticas, e somente uma característica primária distinta, para comparação.
} 


\begin{tabular}{|l|l|}
\hline Atividade comercial & Múltiplos escritórios \\
\hline Localização & Florianópolis, SC \\
\hline Comprimento & 40 \\
\hline Largura & 17,5 \\
\hline Pé-direito & 2,8 \\
\hline Pavimentos & 10 \\
\hline Ano & Década de 90 \\
\hline Vidros & Fumê \\
\hline PAF Norte & $16 \%$ \\
\hline PAF Leste & $35 \%$ \\
\hline PAF Sul & $16 \%$ \\
\hline PAF Oeste & $35 \%$ \\
\hline
\end{tabular}

Quadro 1 - Características gerais do edifício levantado por Santana (2006)

A discussão acerca do entorno do edifício exigiu a criação de mais de um modelo do edifício real, todos válidos para o RTQ-C, e de dois modelos de referência: um sem sombreamento do entorno de acordo com o RTQ-C e um com sombreamento do entorno, o que na prática não é aplicável ao método indicado no regulamento.

A metodologia consiste na apresentação dos programas utilizados na simulação, com foco nos procedimentos para a iluminação, do método de determinação das características do modelo de referência e, em seguida, do método de modelagem das alternativas para simulação. Finalizando, os resultados são apresentados e discutidos. O Quadro 1 apresenta as características gerais do edifício.

\section{Programas de simulação e recursos adotados para a iluminação}

A simulação foi realizada em duas etapas: etapa de simulação do aproveitamento da luz natural, usando o programa DaySim; e etapa de simulação termoenergética, usando o programa EnergyPlus. Ambos utilizam o arquivo climático do tipo TRY de Florianópolis, referente ao ano de 1963.

Os resultados de potência de fato utilizada por um sistema dimerizável fornecidos pelo DaySim foram transferidos para o EnergyPlus como padrão de uso das cargas do sistema de iluminação. Assim, o sistema de iluminação com dimmers não foi realmente modelado no EnergyPlus. A modelagem do sistema de iluminação se resumiu às inserções padrão de um sistema comum, e o padrão de uso das cargas da iluminação regulou a energia instantaneamente consumida por lâmpadas com dimmers.

O procedimento exigiu a modelagem do edifício completo no programa EnergyPlus e a modelagem de cada ambiente em separado no programa DaySim. Além das semelhanças, como dimensões dos ambientes e geometria e reflexões das proteções solares e superfícies internas, foram consideradas as diferenças advindas das reflexões dos edifícios do entorno, o que resultou em diversos modelos para iluminação natural, não apenas devido à orientação solar, mas também devido à posição do ambiente em cada pavimento.

O DaySim permite a inclusão do comportamento do usuário, caracterizando usuários passivos e ativos em alguns recursos do programa, como no controle do ofuscamento. Como o RTQ-C não se refere a um edifício em uso, mas ao nível de eficiência de um "produto", antes da interferência do usuário, optou-se pelas opções passivas, que incluem as proteções solares fixas. Foi utilizada uma malha de pontos de espaçamento de $50 \mathrm{~cm}$ para um sistema com dimmers controlados por fotossensores.

\section{Modelo de referência}

Como citado, o modelo de referência deve ser criado com as características do método prescritivo, mas com características como forma e padrões de uso idênticos aos do modelo do edifício real. Ele deve ser modelado com características específicas para a envoltória, para o sistema de iluminação e para o sistema de condicionamento de ar. Sistemas adicionais que elevam o nível de eficiência do edifício, como aproveitamento da luz natural para redução do consumo pela iluminação artificial, não devem ser incluídos no modelo de referência. 


\section{Envoltória}

A análise pelo método de simulação do RTQ-C foi aplicada visando ao nível de eficiência A para a envoltória. Assim, a equação 2 foi usada para definir as características do modelo de referência para nível A. Conforme indicado no regulamento, deve-se encontrar a maior área de aberturas envidraçadas $\left(\mathrm{PAF}_{\mathrm{T}}\right)$ para o nível de eficiência pretendido usando vidros claros (FS) e ângulos de sombreamento (AHS e AVS) iguais a zero. Além disso, deve-se atender aos pré-requisitos de acordo com o nível de eficiência pretendido. Assim, devese encontrar um $\mathrm{IC}_{\mathrm{env}}$ igual ao limite máximo de IC para o nível A e deve-se adotar os valoreslimite de transmitância térmica e absortância solar.

A Tabela 1 apresenta os dados encontrados para o modelo de referência. $\mathrm{O}$ fator de forma e o fator altura são idênticos aos do modelo do edifício real, e o fator solar e ângulos de sombreamento são fixos. Dessa forma, a variável que se deseja encontrar é o $\mathrm{PAF}_{\mathrm{T}}$, que foi de $17 \%$. O modelo de referência deve também apresentar os valoreslimite de transmitâncias térmicas para coberturas de acordo com o tipo de condicionamento do ambiente diretamente protegido por essas coberturas e de paredes de acordo com a zona bioclimática. Por fim, a absortância solar $(\alpha)$ deve ser 0,4 tanto para paredes quanto para coberturas. Não há iluminação zenital para esse edifício, portanto o pré-requisito de iluminação zenital não foi modelado.

Embora o $\mathrm{PAF}_{\mathrm{T}}$ encontrado seja de $17 \%$, deve-se atentar que esse percentual é distribuído proporcionalmente pela fachada do modelo de referência, em relação ao edifício real. Assim, mantendo as proporções apresentadas nas fachadas norte, leste, sul e oeste da Tabela 1, os PAF de cada fachada do modelo de referência são os indicados na Tabela 2.

\section{Sistema de iluminação}

Os sistemas de iluminação de cada ambiente devem ser modelados usando-se a densidade de potência de iluminação relativa limite, $\mathrm{DPI}_{\mathrm{RL}}$, calculada para cada índice de ambiente (K) encontrado. Caso os índices de ambiente não se encontrem na tabela do RTQ-C, deve-se interpolar a $\mathrm{DPI}_{\mathrm{RL}}$ em função do índice de ambiente encontrado. A Tabela 3 apresenta esses dados para os seis tipos de ambientes de escritório do pavimento-tipo, considerando uma iluminância de 500 lux.

\begin{tabular}{|c|c|c|c|c|c|c|c|}
\hline \multicolumn{4}{|c|}{ Dados para a equação 2} & \multicolumn{4}{|c|}{ Pré-requisitos para nível A } \\
\hline FF & 0,10 & $\mathrm{PAF}_{\mathrm{T}}$ & $0,17(17 \%)$ & $\mathrm{U}_{\mathrm{par}}\left(\mathrm{W} / \mathrm{m}^{2} \mathrm{~K}\right)$ & 3,70 & apar & 0,40 \\
\hline FA & 0,20 & AVS & 0 & $\mathrm{U}_{\mathrm{cob}}\left(\mathrm{W} / \mathrm{m}^{2} \mathrm{~K}\right)$ & 1,00 & acob & 0,40 \\
\hline FS & 0,87 & AHS & 0 & & & & \\
\hline
\end{tabular}

Tabela 1 - Características da envoltória para o modelo de referência

\begin{tabular}{c|c|c|c}
\hline Norte & $9,3 \%$ & Leste & $20,4 \%$ \\
\hline Sul & $9,3 \%$ & Oeste & $20,4 \%$ \\
\hline
\end{tabular}

Tabela 2 - Percentuais de área de abertura envidraçada em cada fachada do modelo de referência

\begin{tabular}{l|c|c|c|c|c|c|c}
\hline Sala & Larg. (m) & Comp. (m) & $\begin{array}{c}\text { Pé-direito } \\
(\mathbf{m})\end{array}$ & $\mathbf{K}$ & $\begin{array}{c}\text { Ilum. } \\
(\mathbf{l u x})\end{array}$ & $\begin{array}{c}\mathbf{D P I}_{\mathbf{R L}} \\
\left(\mathbf{W} / \mathbf{m}^{2} / \mathbf{1 0 0} \mathbf{l u x}\right)\end{array}$ & $\begin{array}{c}\text { DPI } \\
\left(\mathbf{W} / \mathbf{m}^{2}\right)\end{array}$ \\
\hline S11 & 8,75 & 9,00 & 2,80 & 1,58 & 500 & 1,94 & 9,70 \\
\hline S12 & 8,75 & 9,00 & 2,80 & 1,58 & 500 & 1,94 & 9,70 \\
\hline S13 & 22,00 & 7,75 & 2,80 & 2,05 & 500 & 1,88 & 9,40 \\
\hline S14 & 8,75 & 9,00 & 2,80 & 1,58 & 500 & 1,94 & 9,70 \\
\hline S15 & 8,75 & 9,00 & 2,80 & 1,58 & 500 & 1,94 & 9,70 \\
\hline S16 & 22,00 & 7,75 & 2,80 & 2,05 & 500 & 1,88 & 9,40 \\
\hline
\end{tabular}

Tabela 3 - Cargas de iluminação do modelo de referência 


\section{Sistema de condicionamento de ar}

Considerando ser um sistema multisplit, o condicionamento de ar deve ser idêntico ao adotado no modelo do edifício real, porém com coeficientes de desempenho conforme o indicado no método prescritivo para condicionadores de ar não tabelados pelo Inmetro. Para a simulação, não devem ser usados os limites estabelecidos pelo Inmetro mesmo se disponíveis no site do Instituto.

Assim, o nível de eficiência do sistema multisplit do modelo de referência deve ser obtido na tabela 5.1 do RTQ-C, originada da Norma 90.1 (ASHRAE, 2007), referente ao nível de eficiência mínimo de condicionadores a ar para classificação nos níveis A e B. Para a capacidade entre $19 \mathrm{~kW}$ (cerca de 5 TR) e $40 \mathrm{~kW}$ (cerca de 11 TR), o nível de eficiência é expresso pelo coeficiente de desempenho (coefficient of performance, COP), que, para sistemas com aquecimento sem resistência elétrica, é de 2,96 W/W. Esse é o nível de eficiência do sistema, baseado em bombas de calor. Já o restante do sistema de condicionamento de ar do modelo de referência deve ser idêntico ao do modelo do edifício real.

Aqui vale uma observação acerca de sistemas centrais de condicionamento de ar. No caso desses sistemas em modelos de referência de nível A, o modelo de referência deve ainda conter todos os requisitos de projeto do sistema de condicionamento exigidos no método prescritivo para nível A que constam no regulamento, desde que esses requisitos sejam válidos para o clima ou edifício em questão. Como exemplo, um ciclo economizador não deve ser modelado no edifício de referência em climas quentes e úmidos, pois é uma opção economizadora de energia que não funcionaria adequadamente nesses climas.

\section{Modelo do edifício real}

O edifício real foi modelado de forma mais semelhante possível ao descrito por Santana (2006), exceto pelo sistema de condicionamento de ar e pelo sistema de iluminação. Como o edifício empresarial de escritórios contém múltiplas unidades autônomas, cada escritório apresentaria um sistema de condicionamento de ar distinto. Os condicionadores foram uniformizados em todos os ambientes com aparelhos do tipo multisplit com bombas de calor. Já a iluminação foi alterada para as densidades-limite para nível A do regulamento e contendo dimmers, visando à análise do aproveitamento da luz natural.

Foram também modeladas alternativas ao edifício real, denominadas:

(a) entorno: idêntico do modelo real, mas com sombreamento do entorno de acordo com a Figura 2;

(b) brises: idêntico do modelo real, mas com sombreamento por proteções solares de acordo com a Figura 3;

(c) ent/brises: idêntico do modelo real, mas com sombreamento por proteções solares e pelo entorno de acordo com a Figura 4; e

(d) sem luz natural: idêntico do modelo real, mas sem controle dimerizável para aproveitamento da luz natural.

Pelas Figuras 1 a 4, nota-se que as salas laterais são repetidas. Elas poderiam ser unificadas em uma zona térmica apenas. No entanto, elas foram divididas devido à simulação no DaySim, cujas refletâncias das paredes internas são bastante relevantes para a reflexão da luz. Assim, o modelo térmico seguiu o mesmo padrão, embora pudesse ainda ser unificado em uma zona térmica com ajustes no padrão de uso, o que não foi realizado pelo dispêndio de tempo no ajuste numérico do padrão de uso.

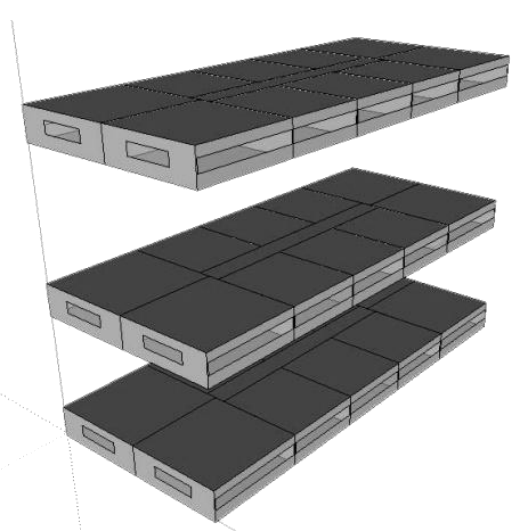

Figura 1 - Edifício real modelado sem sombreamento do entorno e sem proteções solares 


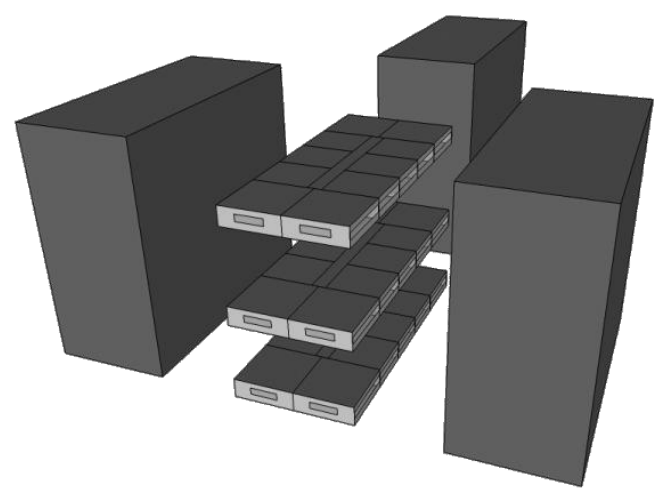

Figura 2 - Edifício real modelado com sombreamento do entorno - vista SE

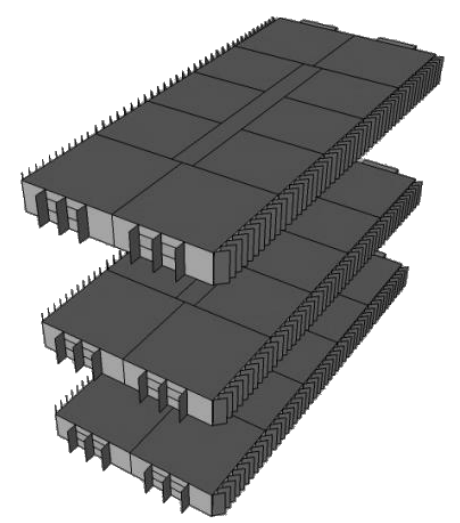

Figura 3 - Edifício real modelado com proteções solares - vista SE

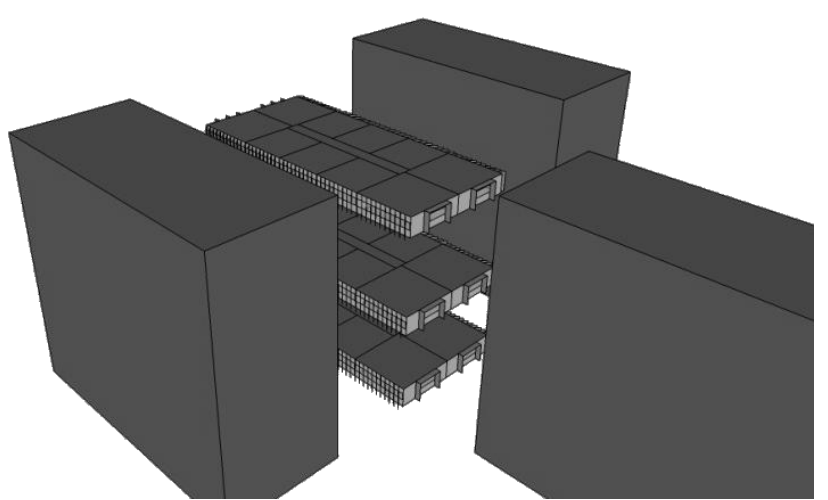

Figura 4 - Edifício real modelado com sombreamento do entorno e proteções solares - vista NE

Já o edifício de referência foi modelado segundo o método de simulação do RTQ-C para nível de eficiência A, e ainda foi modelado um edifício de referência com sombreamento do entorno que não está de acordo com o regulamento, mas que será discutido a seguir.

Os parâmetros dos edifícios modelados são mostrados na Tabela 4 e foram inseridos nos modelos do DaySim e EnergyPlus, de acordo com seus respectivos recursos.

As características físicas do edifício real são de acordo com o levantado por Santana (2009). Essas mesmas características foram modificadas somente se exigido pelo método de simulação do RTQ-C. As cargas, a infiltração e os padrões de uso são típicos para esse uso, exceto as cargas da iluminação, em que foi inserido um sistema de nível de eficiência $\mathrm{A}$, de acordo com o método prescritivo do RTQ-C. Por fim, o sistema de condicionamento de ar é do tipo multisplit, com as eficiências que atualmente são nível D pelo RTQ$\mathrm{C}(2,40 \mathrm{~W} / \mathrm{W}$ para resfriamento e $2,16 \mathrm{~W} / \mathrm{W}$ para aquecimento), mas que são compatíveis com o ano de construção do edifício segundo a Norma 90.1, publicada em 1989 (ASHRAE, 1989) (Tabela 4). 
Os dados analisados foram o consumo anual de eletricidade pela área e os usos finais acarretados por esse consumo. Os usos finais foram divididos em iluminação interna, equipamentos e condicionamento por aquecimento, resfriamento e ventiladores, não devendo os equipamentos ser alterados entre os modelos real e de referência ao aplicar o método de simulação.

\section{Resultados e discussão}

A Tabela 5 e a Figura 5 apresentam os resultados de consumo para os modelos simulados. Pelo método de simulação do RTQ-C, os seis primeiros modelos são válidos, e este artigo ainda discute o sétimo modelo: o modelo de referência sombreado.

Analisando a Tabela 5, vê-se que os maiores usos finais são devidos ao condicionamento de ar por resfriamento, e os menores são devido ao aquecimento, irrelevante para todos os casos considerando edifícios localizados no clima de Florianópolis e com bomba de calor. ${ }^{7}$ Em seguida, o sistema de iluminação apresenta maior participação no consumo anual de energia elétrica dos edifícios, e os equipamentos apresentam consumos idênticos para todos os modelos, como esperado.

O consumo devido ao resfriamento pelo sistema de condicionamento de ar é mais elevado no edifício real sem aproveitamento da luz natural e no edifício real $\left(51,6 \mathrm{kWh} / \mathrm{m}^{2}\right.$ e $48,4 \mathrm{kWh} / \mathrm{m}^{2}$ respectivamente), que não apresentam sombreamento. O consumo mais baixo é o do modelo de referência sombreado $\left(34,9 \mathrm{kWh} / \mathrm{m}^{2}\right)$, seguido do modelo do edifício real com sombreamento do entorno e brises $\left(40,6 \mathrm{kWh} / \mathrm{m}^{2}\right)$.

Já o consumo por iluminação é obviamente mais elevado nos modelos sem aproveitamento da luz natural, tanto no edifício real quanto nos dois modelos de referência $\left(28,1 \mathrm{kWh} / \mathrm{m}^{2}\right)$. Ao analisar os consumos por iluminação com aproveitamento da luz natural, vê-se que a alternativa mais econômica é o modelo do edifício real com brises, cujo sistema de iluminação alcança somente 21,6 $\mathrm{kWh} / \mathrm{m}^{2}$, mais baixo até que o modelo do edifício real sem sombreamento, devido à reflexão das proteções solares. $\mathrm{O}$ modelo real com sombreamento do entorno também apresenta consumos baixos, de $24,1 \mathrm{kWh} / \mathrm{m}^{2}$, menores que o modelo real com sombreamento do entorno e ainda com brises. Este último modelo apresenta um sombreamento excessivo, que se reflete na economia pelo sistema de iluminação, que não compensa o baixo consumo por resfriamento no total anual do edifício.

Ao verificar os consumos totais, vê-se que o modelo do edifício real sem aproveitamento da luz natural e o modelo do edifício real, mesmo com aproveitamento da luz natural, não alcançam nível A, pois seus consumos são, respectivamente, 93,2 $\mathrm{kWh} / \mathrm{m}^{2}$ e $102,9 \mathrm{kWh} / \mathrm{m}^{2}$, mais elevados que o limite máximo para o nível $\mathrm{A}$ do modelo de referência, de $93,0 \mathrm{kWh} / \mathrm{m}^{2}$. Mas se o simulador optar pelo sombreamento do entorno, esse mesmo edifício torna-se nível de eficiência $\mathrm{A}$, pois seu consumo se reduz para $88,6 \mathrm{kWh} / \mathrm{m}^{2}$. Essa opção aproxima-se de um edifício com brises, que, nesse caso, alcançou um consumo total anual de 85,1 $\mathrm{kWh} / \mathrm{m}^{2}$. Já se o edifício com entorno sombreado apresentar brises, seu sombreamento excessivo provoca um consumo de $91,1 \mathrm{kWh} / \mathrm{m}^{2}$, devido à já comentada economia irrelevante do sistema de iluminação. Vê-se, então, como os brises são dispensáveis nessa situação.

Até este momento, a análise ocorreu de acordo com o RTQ-C. Caso fosse permitido que o modelo de referência fosse sombreado quando edifícios com sombreamento do entorno fossem simulados nenhum dos modelos teria nível de eficiência A. O modelo de referência sombreado apresenta consumo de $86,0 \mathrm{kWh} / \mathrm{m}^{2}$, e somente o modelo do edifício real com os brises apresenta consumo anual de eletricidade mais baixo que o modelo real sombreado. Ou seja, nenhum dos modelos com sombreamento do entorno é capaz de chegar ao nível de eficiência A.

\footnotetext{
${ }^{7}$ Um sistema split com aquecimento por resistência elétrica gera um consumo total de $134,6 \mathrm{kWh} / \mathrm{m}^{2} \mathrm{no}$ modelo do edifício real, sendo o aquecimento de 6,0 $\mathrm{kWh} / \mathrm{m}^{2}$, e o resfriamento de $78,5 \mathrm{kWh} / \mathrm{m}^{2}$, enquanto no modelo do edifício real com brises o consumo total é de $119,1 \mathrm{kWh} / \mathrm{m}^{2}$, sendo que o aquecimento alcanca 6,7 $\mathrm{kWh} / \mathrm{m}^{2}$, e o resfriamento, $65,2 \mathrm{kWh} / \mathrm{m}^{2}$. Esse tipo de condicionamento não é considerado eficiente e, portanto, não foi tratado como relevante no desenvolvimento do método de simulação.
} 


\begin{tabular}{|c|c|c|c|c|c|c|c|}
\hline \multirow[b]{2}{*}{ Parâmetro } & \multicolumn{5}{|c|}{ Modelo do edifício real } & \multicolumn{2}{|c|}{ Modelo de referência } \\
\hline & Real & Entorno & Brises & $\begin{array}{c}\text { Entorno/ } \\
\text { brises }\end{array}$ & $\begin{array}{c}\text { Sem luz } \\
\text { natural }\end{array}$ & RTQ-C & Entorno \\
\hline Dimensões (m) & $17 \times 40$ & $17 \times 40$ & $17 \times 40$ & $17 \times 40$ & $17 \times 40$ & $17 \times 40$ & $17 \times 40$ \\
\hline Número pav. & 10 & 10 & 10 & 10 & 10 & 10 & 10 \\
\hline Pé-direito (m) & 2,8 & 2,8 & 2,8 & 2,8 & 2,8 & 2,8 & 2,8 \\
\hline $\begin{array}{l}\text { Orientação das } \\
\text { maiores fachadas }\end{array}$ & $\begin{array}{l}\text { leste- } \\
\text { oeste }\end{array}$ & $\begin{array}{l}\text { leste- } \\
\text { oeste }\end{array}$ & $\begin{array}{l}\text { leste- } \\
\text { oeste }\end{array}$ & $\begin{array}{l}\text { leste- } \\
\text { oeste }\end{array}$ & $\begin{array}{l}\text { leste- } \\
\text { oeste }\end{array}$ & $\begin{array}{l}\text { leste- } \\
\text { oeste }\end{array}$ & $\begin{array}{l}\text { leste- } \\
\text { oeste }\end{array}$ \\
\hline $\mathrm{PAF}_{\mathrm{t}}$ & $29 \%$ & $29 \%$ & $29 \%$ & $29 \%$ & $29 \%$ & $18 \%$ & $18 \%$ \\
\hline $\mathrm{U}_{\mathrm{par}}\left(\mathrm{W} / \mathrm{m}^{2} \mathrm{~K}\right)$ & 2,01 & 2,01 & 2,01 & 2,01 & 2,01 & 3,7 & 3,7 \\
\hline $\mathrm{U}_{\mathrm{cob}}\left(\mathrm{W} / \mathrm{m}^{2} \mathrm{~K}\right)$ & 0,51 & 0,51 & 0,51 & 0,51 & 0,51 & 1,0 & 1,0 \\
\hline$\alpha_{\text {par }}$ & 0,6 & 0,6 & 0,6 & 0,6 & 0,6 & 0,4 & 0,4 \\
\hline$\alpha_{\mathrm{cob}}$ & 0,7 & 0,7 & 0,7 & 0,7 & 0,7 & 0,4 & 0,4 \\
\hline Brises & - & - & Sim & $\operatorname{sim}$ & - & - & - \\
\hline $\begin{array}{l}\text { Iluminação média } \\
\text { esc. }\left(\mathrm{W} / \mathrm{m}^{2}\right)\end{array}$ & 9,69 & 9,69 & 9,69 & 9,69 & 9,69 & 9,69 & 9,69 \\
\hline $\begin{array}{l}\text { Iluminação média } \\
\left(\mathrm{W} / \mathrm{m}^{2}\right)\end{array}$ & 10,30 & 10,30 & 10,30 & 10,30 & 10,30 & 10,30 & 10,30 \\
\hline $\begin{array}{l}\text { Controle de } \\
\text { iluminação esc. }\end{array}$ & dimmer & dimmer & dimmer & dimmer & - & - & - \\
\hline $\begin{array}{l}\text { Equipamentos } \\
\left(\mathrm{W} / \mathrm{m}^{2}\right)\end{array}$ & 9,7 & 9,7 & 9,7 & 9,7 & 9,7 & 9,7 & 9,7 \\
\hline $\begin{array}{l}\text { Ocupação } \\
(\text { pessoas/m²) }\end{array}$ & 0,15 & 0,15 & 0,15 & 0,15 & 0,15 & 0,15 & 0,15 \\
\hline $\begin{array}{l}\text { Padrão de uso } \\
\text { geral }\end{array}$ & $8 \mathrm{~h}$ a $18 \mathrm{~h}$ & $8 \mathrm{~h}$ a $18 \mathrm{~h}$ & $8 \mathrm{~h}$ a $18 \mathrm{~h}$ & $8 \mathrm{~h}$ a $18 \mathrm{~h}$ & $8 \mathrm{~h}$ a $18 \mathrm{~h}$ & $8 \mathrm{~h}$ a $18 \mathrm{~h}$ & $8 \mathrm{~h}$ a $18 \mathrm{~h}$ \\
\hline Infiltração & $1 \mathrm{ACH}$ & $1 \mathrm{ACH}$ & $1 \mathrm{ACH}$ & $1 \mathrm{ACH}$ & $1 \mathrm{ACH}$ & $1 \mathrm{ACH}$ & $1 \mathrm{ACH}$ \\
\hline $\begin{array}{l}\text { Condicionamento } \\
\text { de ar }\end{array}$ & mulisplit & mulisplit & mulisplit & mulisplit & mulisplit & mulisplit & Mulisplit \\
\hline $\begin{array}{l}\text { Eficiência (COP, } \\
\text { W/W) }\end{array}$ & $\begin{array}{c}2,40 \mathrm{e} \\
1,80\end{array}$ & $\begin{array}{c}2,40 \mathrm{e} \\
1,80\end{array}$ & $\begin{array}{c}2,40 \mathrm{e} \\
1,80\end{array}$ & $\begin{array}{c}2,40 \mathrm{e} \\
1,80\end{array}$ & $\begin{array}{c}2,40 \mathrm{e} \\
1,80\end{array}$ & $\begin{array}{c}3,02 \mathrm{e} \\
2,26\end{array}$ & $\begin{array}{c}3,02 \mathrm{e} \\
2,26\end{array}$ \\
\hline T set point $\left({ }^{\circ} \mathrm{C}\right)$ & 18 e 24 & 18 e 24 & 18 e 24 & 18 e 24 & 18 e 24 & 18 e 24 & 18 e 24 \\
\hline $\begin{array}{l}\text { Sombreamento } \\
\text { do entorno }\end{array}$ & - & $\begin{array}{c}3 \text { blocos a } \\
12 \mathrm{~m} \\
\text { cada, de } \\
17 \times 40 \mathrm{x} \\
35 \mathrm{~m}\end{array}$ & & $\begin{array}{c}3 \text { blocos a } \\
12 \mathrm{~m} \\
\text { cada, de } \\
17 \times 40 \mathrm{x} \\
35 \mathrm{~m}\end{array}$ & - & 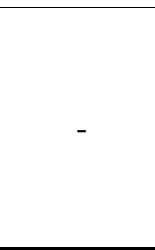 & $\begin{array}{c}3 \text { edifícios } \\
\text { a } 12 \mathrm{~m} \\
\text { cada, de } \\
17 \text { x } 40 \mathrm{x} \\
35 \mathrm{~m}(\mathrm{x}, \mathrm{y} \\
\text { e z) }\end{array}$ \\
\hline
\end{tabular}

Tabela 4 - Características dos modelos simulados

\begin{tabular}{l|c|c|c|c|c|c|c}
\hline \multirow{2}{*}{$\begin{array}{l}\text { Uso final } \\
\left(\mathbf{k W h} / \mathbf{m}^{2}\right)\end{array}$} & Real & $\begin{array}{c}\text { Real } \\
\text { entorno }\end{array}$ & $\begin{array}{c}\text { Real } \\
\text { brises }\end{array}$ & $\begin{array}{c}\text { Real } \\
\text { brises } \\
\text { entorno }\end{array}$ & $\begin{array}{c}\text { Sem luz } \\
\text { natural }\end{array}$ & $\begin{array}{c}\text { Referência } \\
\text { nível A }\end{array}$ & $\begin{array}{c}\text { Referência } \\
\text { nível A } \\
\text { entorno }\end{array}$ \\
\cline { 2 - 8 } & 93,2 & 88,6 & 85,1 & 91,1 & 102,9 & 93,0 & 86,0 \\
\hline Edifício & 48,4 & 41,9 & 41,0 & 40,6 & 51,6 & 41,4 & 34,9 \\
\hline Resfriamento & 0,32 & 0,36 & 0,35 & 0,35 & 0,31 & 0,36 & 0,41 \\
\hline Aquecimento & 1,39 & 0,93 & 0,86 & 0,84 & 1,55 & 1,81 & 1,21 \\
\hline Ventiladores & 21,7 & 24,1 & 21,6 & 28,0 & 28,1 & 28,1 & 28,1 \\
\hline Equipaminação & 21,3 & 21,3 & 21,3 & 21,3 & 21,3 & 21,3 & 21,3 \\
\hline
\end{tabular}

Tabela 5 - Consumo anual de eletricidade do edifício e seus usos finais em $\mathrm{kWh} / \mathrm{m}^{2}$ 


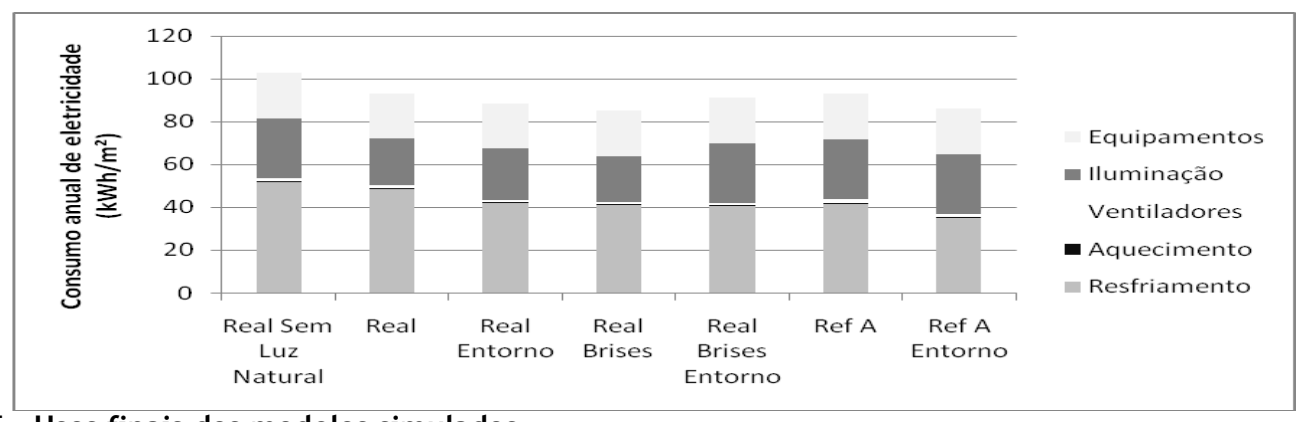

Figura 5 - Usos finais dos modelos simulados

Assim, sombreamento do entorno é visto como uma forma de substituição dos brises, com chances de garantir o nível de eficiência A pelo método de simulação. $O$ excessivo sombreamento causado pela combinação "entorno e brises" pode elevar o consumo de energia, e o modelo de referência se descaracteriza de seu objetivo caso o sombreamento do entorno seja exigido quando ele existe no modelo do edifício real.

\section{Conclusão}

Este artigo apresentou a aplicação do método de simulação do Regulamento Técnico da Qualidade do Nível de Eficiência Energética de Edifícios Comerciais, de Serviços e Públicos, RTQ-C (BRASIL, 2009), com destaque para uma discussão acerca do sombreamento do entorno. O método de avaliação foi discutido abordando-se os programas de simulação, a modelagem e a opção de sombrear ou não o edifício usando os edifícios localizados em seu entorno imediato, método prescritivo e método de simulação.

O método de simulação proporciona a flexibilidade necessária para realizar compensações entre sistemas mais e menos eficientes, não exige parâmetros de projeto de condicionamento de ar predefinidos e integra as bonificações no nível de eficiência energética geral do edifício.

Foram simuladas alternativas de um edifício real em Florianópolis com e sem sombreamento do entorno e com e sem proteções solares por brises. Estes foram comparados ao modelo de referência segundo o método de simulação do RTQ-C, integrando o aproveitamento da luz natural para economia de energia elétrica pelo sistema de iluminação artificial. Mostrou-se que o modelo de referência não deve ser sombreado, pois seu consumo anual, de $93,0 \mathrm{kWh} / \mathrm{m}^{2}$, é reduzido para $86,0 \mathrm{kWh} / \mathrm{m}^{2}$ quando essa variável é incluída no modelo de referência. Pelas exigências do regulamento, nenhum dos modelos testados alcançaria nível A caso o sombreamento causado pelo entorno fosse incluído no modelo de referência, visto que o único modelo que alcançou um consumo inferior apresenta apenas brises (sem sombreamento do entorno). Além disso, esse sombreamento no edifício de referência descaracterizaria o modelo em seus objetivos modelo de referência para comparação com outros casos cujas características primárias são comuns e, ainda, essa opção poderia estimular o sombreamento excessivo no edifício, prejudicando o resultado final por reduzir sua eficiência.

Espera-se que as questões discutidas esclareçam a origem dos critérios adotados para o desenvolvimento do método de simulação do RTQ-C e indiquem seu potencial de uso de acordo com as características do edifício a ser avaliado, do tempo disponível para a avaliação e dos recursos existentes.

\section{Referências}
AMERICAN SOCIETY OF HEATING, REFRIGERATING AND AIR CONDITIONING ENGINEERS. Standard 90.1-1989: energy standard for buildings except low-rise residential buildings. Atlanta, 1989.
AMERICAN SOCIETY OF HEATING, REFRIGERATING AND AIR CONDITIONING ENGINEERS. Standard 90.1-2007: energy standard for buildings except low-rise residential buildings. Atlanta, 2007.
AMERICAN SOCIETY OF HEATING, REFRIGERATING AND AIR CONDITIONING ENGINEERS. Standard 140-2004: standard method of test for the evaluation of building energy analysis computer programs. Atlanta, 2004.
BRASIL. Instituto Nacional de Metrologia, Normalização e Qualidade Industrial (INMETRO). Portaria 163, de 08 de junho de 2009.
Regulamento Técnico da Qualidade do Nível de Eficiência Energética de Edifícios Comerciais, de Serviços e Públicos. Rio de Janeiro, 2009.
Disponível em:
<http://www.inmetro.gov.br/legislacao/rtac/pdf/RT AC001462.pdf >. Acesso em: 03 mar. 2009. 
CARLO, J. C. Desenvolvimento de Metodologia de Avaliação da Eficiência Energética do Envoltório de Edificações Não-Residenciais. 2008. 196 f. Tese (Doutorado em Engenharia Civil) - Centro Tecnológico, Universidade Federal de Santa Catarina Florianópolis, 2008.

CARLO, J.; LAMBERTS, R. Development of Envelope Efficiency Labels for Commercial Buildings: effect of different variables on electricity consumption. Energy and Buildings, Lausanne, v. 40, n. 11, p. 2002-2008, 2008.

\section{COMMISSION INTERNATIONALE DE}

L'ECLAIRAGE (CIE). CIE 171:2006: test cases to assess the accuracy of lighting computer programs. Paris, 2006.

CRAWLEY, D. B., et al. EnergyPlus: creating a new-generation building energy simulation. In: Energy and Buildings, Lausanne, v. 22, p. 319331, 2001.

GONÇALVES, H. E.; ALMEIDA, F. O Uso de Programas de Simulação Dinâmica de Edifícios em Estudos de Sensibilidades. In: ENCONTRO NACIONAL DE MODELOS DE SIMULAÇÃO DE AMBIENTES, 1995, São Paulo. Anais... São Paulo: ENMSA, 1995. p. 231-245.

GU, L. Airflow Nerwork Modeling in EnergyPlus. In: IBPSA BUILDING SIMULATION CONFERENCE AND EXHIBITION, 2007, Beijing, Proceedings... Beijing: IBPSA, 2007. p. 964-971.

HAVES, P. New Tools for Building Simulation. In: CONGRESSO DE AR CONDICIONADO, REFRIGERAÇÃO, AQUECIMENTO E VENTILAÇÃO DO MERCOSUL, 4., 2004, Curitiba. Anais... Curitiba: ASBRAV, 2004. 1 CD-ROM.

JUDKOFF, R. Final Task Management Report: testing and validation of building energy simulation tools. Colorado: International Energy Agency and Solar Heating \& Cooling Programme, 2008.

JUDKOFF, R.; NEYMARK, J. What Did They do in IEA 34/43? Or How To Diagnose and Repair Bugs in 500,000 Lines of Code. In:

INTERNATIONAL IBPSA CONFERENCE, 6 ., 2009, Glasgow. Proceedings... Glasgow: IBPSA, 2009. p. 1091-1098.

LOUTZENHISER, P.; MANZ, H.; MAXWELL, G. Empirical Validations of Shading/Daylighting/Load Interactions in Building Energy Simulation Tools: a report for the International Energy Agency's SHC Task 34/ECBCS Annex 43 Project C. Sydney: International Energy Agency's, 2007.
MAHDAVI, A. Reflections on Computational Building Models. Building and Environment, Oxford, v. 39, p. 913-925, 2004.

MANZ, H., et al. Series of Experiments for Empirical Validation of Solar Gain Modeling in Building Energy Simulation Codes: experimental setup, test cell characterization, specifications and uncertainty analysis. Building and Environment, Oxford, v. 41, n. 12, p. 1784-1797, 2006.

PEREIRA, R. C., et al. Metodologia para Avaliação de Ferramentas de Simulação da Iluminação Natural através de Mapeamento Digital de Luminâncias. In: ENCONTRO NACIONAL DE CONFORTO NO AMBIENTE CONSTRUÍDO, 9., 2009, Ouro Preto. Anais... Porto Alegre: ANTAC, 2007. p. 1431-1440.

PROCEL. Manual para Aplicação dos Regulamentos: RTQ-C e RAC-C. Rio de Janeiro: Procel/Eletrobras, 2009. Disponível em: <http://www.labeee.ufsc.br/eletrobras/etiquetagem /downloads.php>. Acesso em: 25 nov. 2009

RAMOS, G.; GHISI, E. Comparação de Iluminâncias Internas e Externas Obtidas por Meio de Simulação Computacional. In: ENCONTRO NACIONAL DE TECNOLOGIA NO AMBIENTE CONSTRUÍDO, 8., 2008, Fortaleza. Anais... Porto Alegre: ANTAC, 2008.

RAMOS, G.; GHISI, E. Avaliação do Cálculo da Iluminação Natural Realizada pelo Programa Energyplus. In: ENCONTRO NACIONAL DE CONFORTO NO AMBIENTE CONSTRUÍDO, 9., 2009, Natal. Anais... Porto Alegre: ANTAC, 2009. p. 1261-1270.

REINHART, C. F.; HERKEL S. The Simulation of Annual Daylight Illuminance Distributions: a state-of-the-art comparison of six RADIANCEbased methods. Energy and Buildings, Lausanne, v. 32, p. 167-187, 2000.

REINHART, C. F.; MARDALJEVIC, J.; ROGERS, Z. Dynamic Daylight Performance Metrics for Sustainable Building Design. Leukos, v. 3, n. 1, p. 1-25, 2006.

SANTANA, M. V. Influência de Parâmetros Construtivos no Consumo de Energia de Edifícios de Escritório Localizados em Florianópolis-SC. 2006. 182 f. Dissertação (Mestrado em Engenharia Civil) - Universidade Federal de Santa Catarina, Florianópolis, 2006.

\section{Agradecimentos}

Ao Procel Edifica/Eletrobrás, pelo financiamento da pesquisa. 